\title{
Amplificação e clonagem de pulsos curtos em am- plificadores ópticos paramétricos a fibra óptica.
}

\author{
A. V. Nascimento \\ Escola Politécnica de Pernambuco \\ Universidade de Pernambuco \\ 50.720-001 - Recife, Brasil \\ allisson_vieira@hotmail.com
}

\author{
João F. L. de Freitas \\ Escola Politécnica de Pernambuco \\ Universidade de Pernambuco \\ 50.720-001 - Recife, Brasil \\ joaoflf@poli.br
}

Resumo Pulsos curtos são amplamente utilizados nas telecomunicações ópticas por suas características essenciais para sistema de comunicações ópticos em taxas de transmissão de Gbits/s. Assim, estudamos a amplificação de pulsos de 5 ps em um amplificador óptico paramétrico via simulação computacional.

\footnotetext{
Abstract Short pulses are widely used in optical telecommunications for their essential characteristics to optical communication system at data rates of Gbits/s. Thus, we have studied the amplification of pulses of 5 ps in an optical parametric amplifier throughcomputer simulation.
} 


\section{Introdução}

O texto começa aqui. Títulos de seções devem usar o estilo "Titulo" do menu de estilos. Para o texto use o estilo "Body Text" (Times 10). Referências devem ser chamadas no texto usando números [2,3], e, caso os autores assim desejarem, por exemplo, de acordo com Freire [4].

O desenvolvimento das modernas telecomunicações ópticas (CO) deve-se principalmente aos estudos de efeitos de propagação de luz em fibras ópticas, os quais podem ser classificados como lineares (EL) e não lineares (ENL) $[1,2,3,4,5]$.

A resposta de um meio a passagem de uma onda eletromagnética é representada pela polarização elétrica. Esta resposta se processa em vários níveis. Quando a polarização elétrica for linear com a amplitude do campo eletromagnético dizemos que esta resposta é um efeito linear, e quando a polarização é proporcional ao quadrático ou de ordem maior com a amplitude do campo podemos dizer que o efeito é não linear $[1,2,3]$. A principal consequência desta definição é que os EL não geram novas frequências ópticas durante a propagação, enquanto os ENL são caracterizados pela geração de novas componentes de frequência durante a propagação do campo eletromagnético no guia de onda. Os ENL ocorrem por uma resposta não linear da polarização eletromagnética, isto se deve a dois fatores ou sua combinação:

\section{O campo tem intensidade alta;}

2. O meio é composto por materiais que possuem alta resposta não linear, Por exemplo, fibras ópticas de sílica dopadas com germânio; fibras ópticas dopadas com Érbio.

De um modo geral, podemos observar ENL como uma mudança no índice de refração do meio como uma função da intensidade óptica.

Dentre os principais EL: atenuação, dispersão de modos de propagação, dispersão de modos de polarização e cromática [1].

Dentre os principais ENL: auto-modulação de fase AMF, modulação de fase cruzada MFC,espalhamento Brillouin estimulado, espalhamento Raman estimulado, mistura de quatro ondas FWM. Instabilidade modulacional, conversão ascendente de frequência [1,2].

ENL são os principais limitadores das taxas de comunicação e do tamanho dos enlaces totalmente ópticos, bem como, podem ser usados no desenvolvimento de dispositivos ópticos, como por exemplo: Amplificadores ópticos, conversores de comprimento de onda, lasers pulsados e $\mathrm{CW}$ e etc $[3,4]$.
Dentro dos ENL nos chama a atenção o efeito de mistura de quatro ondas que é a base para construção e desenvolvimento de amplificadores ópticos paramétricos a fibra (FOPA) $[1,2,3]$. Veja na figura 1 um diagrama idealizado de um FOPA. Estudos de amplificadores ópticos são abundantes na literatura [6-13], estes amplificadores são amplamente utilizados em diversas situações em comunicações ópticas [1].

Pulsos curtos (1-50 ps) são essenciais em sistemas de comunicações ópticas de alto desempenho (Gbits/s), por exemplo para pulsos com bit slots de 10 ps ( $100 \mathrm{Gbits} / \mathrm{s})$ temos que ter pulsos de no máximo 5 ps. Assim estudarmos as mudanças do seu perfil temporal ao longo de um meio ganho é essencial para uma definição da qualidade do amplificador.

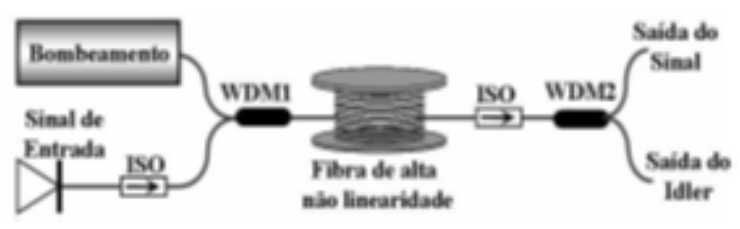

Fig.1: Montagem experimental de um FOPA, WDM1 acoplasinal e bombeamento, WDM2 desacopla sinal e idler e remove bombeamento residual, ISO são isoladores ópticos.

Neste trabalho, desenvolvemos, via simulação computacional estudos sobre amplificação e clonagem de pulsos de 5 ps em FOPA.

\section{Modelagem computacional}

A simulação computacional da amplificação e clonagem de pulsos em amplificadores ópticos paramétricos a fibra óptica pode ser obtida mediante a resolução da equação não linear de Schrödinger. Esta equação se caracteriza por traduzir perfeitamente os efeitos lineares e não lineares presentes na propagação da luz na fibra óptica e é definida como:

$$
\frac{\partial A}{\partial z}=-\frac{\alpha A}{2}-\frac{i \beta_{2}}{2} \frac{\partial^{2} A}{\partial T^{2}}+\frac{\beta_{3}}{6} \frac{\partial^{3} A}{\partial T^{3}}+i \gamma|A|^{2} A
$$

onde: $A$ - é a amplitude transversal do campo elétrico (envoltória do pulso), y - é o parâmetro de não linearidade, $\beta_{i}$ - é a intensidade dos osciladores que participam da i-ésima ressonância (parâmetro responsável pela dispersão cromática) e $\alpha$ - parâmetro de atenuação. 
Analisamos o comportamento dos sinais no meio, resolvendo numericamente a equação de propagação utilizando técnicas numéricas tais como, Split-Step Fourier $[1,4]$ e Diferenças finitas.

O Split-Step (Fourier), em análise numérica, é um método pseudo-espectral. Tal método numérico é utilizado para resolver equações diferenciais parciais, a exemplo da equação não- linear de Schrödinger. Utilizamos esse método, haja vista que nas equações de propagação de luz em fibras ópticas ocorre a interação de mecanismos lineares e não lineares, o que difículta encontrar soluções analíticas gerais.

Tanto a parte linear como a não linear, isoladas, têm soluções analíticas. Todavia, a equação não linear de Schrödinger composta desses dois termos não tem uma solução analítica geral. Assim, se apenas um pequeno passo " $h$ " é tomado ao longo de " $z$ ", as duas partes podem ser tratadas separadamente com apenas um pequeno erro "numérico".

Após a aplicação desse método numérico à equação não linear de Schrödinger (1) encontramos uma equação referente aos mecanismos lineares (Parte Linear) e outra aos não lineares (Parte Não Linear), são elas:

1. Parte Linear: equação complexa que pode ser desmembrada em sua parte real e imaginária da seguinte forma:

- Parte real:

$$
R\left[\tilde{A}\left(z+h, \omega^{\prime}\right)\right]=R\left[\tilde{A}_{j}(z)\right] \mathrm{e}^{\frac{-\alpha h}{2}} \cos (\phi)+I\left[\tilde{A}_{j}(z)\right] \mathrm{e}^{\frac{-\alpha h}{2}} \operatorname{sen}(\phi)
$$

- Parte imaginária:

$I\left[\tilde{A}\left(z+h, \omega^{\prime}\right)\right]=I\left[\tilde{A}_{j}(z)\right] \mathrm{e}^{\frac{-\alpha h}{2}} \cos (\phi)-R\left[\left[\tilde{A}_{j}(z)\right] \mathrm{e}^{\frac{-\alpha h}{2}} \operatorname{sen}(\phi)\right.$

onde: $\square$ - é definido como:

$$
\phi=\left[\frac{-\beta_{2 j}}{2}\left(\omega_{j}^{2}+\omega^{2}\right)+\frac{\beta_{3 j}}{6}\left(\omega^{3}+3 \omega_{j}^{\prime} \omega^{2}+3 \omega_{j}^{2} \omega+\omega_{j}^{\prime 3}\right)\right] h
$$

2. Parte Não linear: Também é uma equação complexa que pode ser separada em sua parte real e imaginária da seguinte forma:

- Parte real:

$$
R\left[A_{j}(z+h)\right]=R\left[A_{j}(z)\right] \mathrm{e}^{\phi_{1}} \cos \left(\phi_{2}\right)-I\left[A_{j}(z)\right] \mathrm{e}^{\phi_{1}} \sin \left(\phi_{2}\right)
$$

$$
I\left[A_{j}(z+h)\right]=R\left[A_{j}(z)\right] \mathrm{e}^{\phi_{1}} \sin \left(\phi_{2}\right)+I\left[A_{j}(z)\right] \mathrm{e}^{\phi_{i}} \cos \left(\phi_{2}\right)
$$

onde : $\Phi 1$ e $\Phi 2$ - são definidos como:

$$
\begin{aligned}
& \phi_{1}=\frac{\gamma P_{B} \mathrm{e}^{-\alpha z} h \cos \left(2 \gamma P_{B} z\right)\left[R\left(A_{m}\right) I\left(A_{j}\right)+R\left(A_{j}\right) I\left(A_{m}\right)\right]}{\left|A_{j}\right|^{2}} \\
& \frac{+\gamma P_{B} \mathrm{e}^{-\alpha z} h \sin \left(2 \gamma P_{B} z\right)\left[I\left(A_{m}\right) I\left(A_{j}\right)-R\left(A_{m}\right) R\left(A_{j}\right)\right]}{\left|A_{j}\right|^{2}} \\
& \phi_{2}=\gamma h\left(2 P_{B} \mathrm{e}^{-\alpha z}+2\left|A_{m}\right|^{2}+\left|A_{j}\right|^{2}\right) \\
& \frac{+\gamma P_{B} \mathrm{e}^{-\alpha z} h \cos \left(2 \gamma P_{B} z\right)\left[R\left(A_{m}\right) R\left(A_{j}\right)-I\left(A_{m}\right) I\left(A_{j}\right)\right]}{\left|A_{j}\right|^{2}} \\
& \frac{+\gamma P_{B} \mathrm{e}^{-\alpha z} h \sin \left(2 \gamma P_{B} z\right)\left[R\left(A_{m}\right) I\left(A_{j}\right)+R\left(A_{j}\right) I\left(A_{m}\right)\right]}{\left|A_{j}\right|^{2}}
\end{aligned}
$$

O delineamento mostrado acima exibe os passos algébricos necessários para obtermos as equações base para criação do algoritmo de simulação de pulsos curtos. Em nosso caso, optamos por programação em linguagem $\mathrm{C} / \mathrm{C}++$.

\section{Pulsos Curtos}

As atuais pesquisas técnicas e científicas das diversas áreas das telecomunicações têm em comum o objetivo de transmitir um maior número de informações com menor perda de dados. Os resultados obtidos da propagação de pulsos curtos em FOPA mostraram-se uma medida eficaz e muito vantajosa para tal finalidade.

Definem-se pulsos curtos como aqueles cuja largura a meia altura do pulso encontra-se na faixa de 1-50 ps. Essa característica em telecomunicações ópticas promove uma maior transmissão de dados em um menor 'bit-slot' e isso significa um grande avanço nas comunicações.

As figuras 3.1 e 3.2 mostram a amplificação do sinal e do "idler" em guias de ondas cilíndricos com uma largura a meia altura igual a $5 \mathrm{ps}$. Assim, podemos por exemplo criar bit slots de 10 ps (taxas de transmissão de $100 \mathrm{Ghz}$ por canal óptico) o que nos fornece possibilidades de taxas de transmissão de bits maiores que o atual estado da arte (40 a $80 \mathrm{Ghz}$ ). Na figura 3.1 exibe-se a amplificação de aproximadamente $21 \mathrm{~dB}$ de um pulso curto de 5 os com perfil de entrada sech e comprimento de onda em $1549 \mathrm{~nm}$ ao longo de uma fibra de $500 \mathrm{~m}$ de alta não linearidade.

- Parte imaginária: 


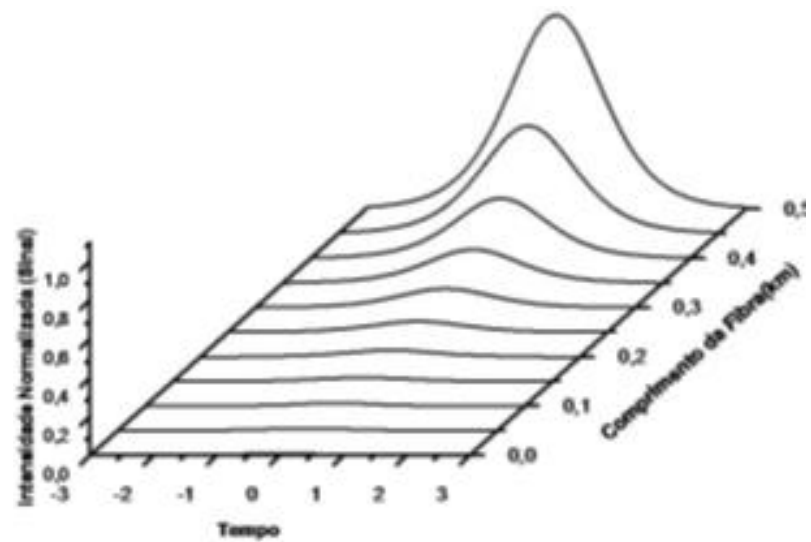

Fig. 3.1: Sinal amplificado através de um amplificador óptico paramétrico (FOPA).

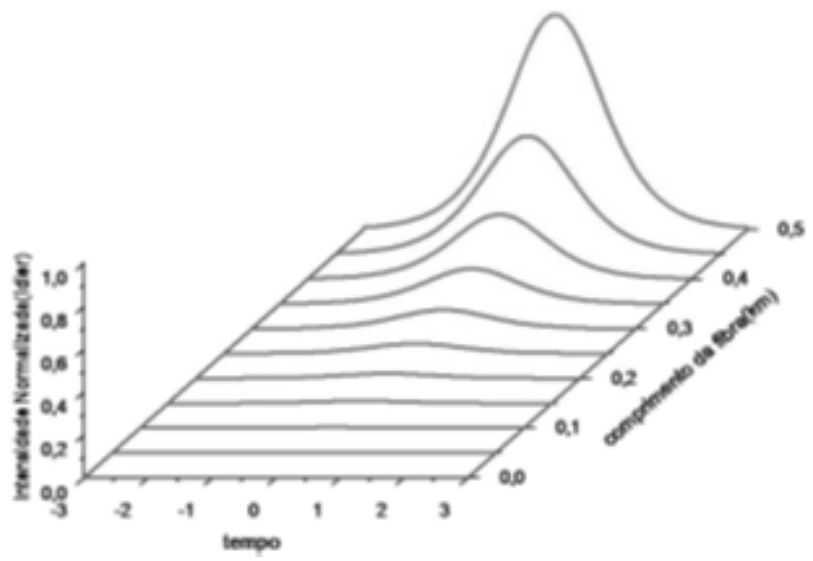

Fig. 3.2: Idler amplificado através da clonagem de pulsos.

A figura 3.3 mostra a intensidade do pulso amplificada de forma normalizada. Percebe-se, portanto, que não há geração de novas frequências, o que é um excelente resultado pois dessa forma não existe distorção no perfil temporal do pulso original ao longo da linha de amplificação.

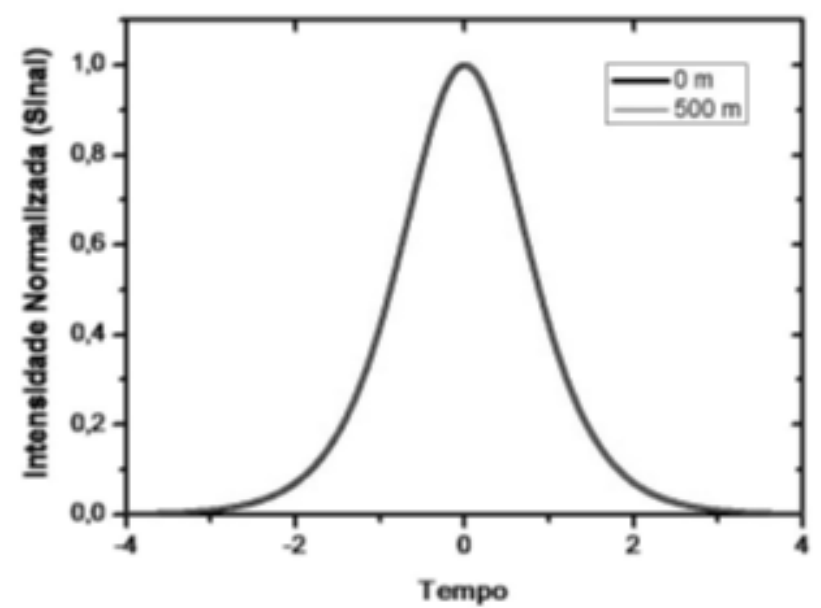

Fig. 3.3: Amplificação do Sinal (5ps)-Potência Normalizada.

\subsection{Clonagem de Pulsos}

Os resultados mostram também o perfeito casamento de fase ocorrido na propagação, o qual proporcionou o surgimento de um pulso de luz em um outro comprimento de onda (Idler), ou seja, ocorreu uma clonagem do sinal . Para as telecomunicações ópticas, tal fato é extremamente desejável, tendo em vista que dessa forma é possível a conversão de sinais em comprimentos de onda distintos.

A figura 3.2 exibe a clonagem do pulso curto de 1549 $\mathrm{nm}$ em um pulso amplificado de $1521 \mathrm{~nm}$ com uma eficiência de conversão de aproximadamente $21 \mathrm{~dB}$. As figuras 3.3 e 3.4, mostram a amplificação do sinal e do idler, respectivamente, durante a propagação do sinal na fibra. Comparando-as percebesse que o idler inicialmente tem uma potência constante muito baixa da ordem de 10-12 (curca azul da figura 3.4) e esta é amplificada juntamente com o sinal(figura 3.3 ).

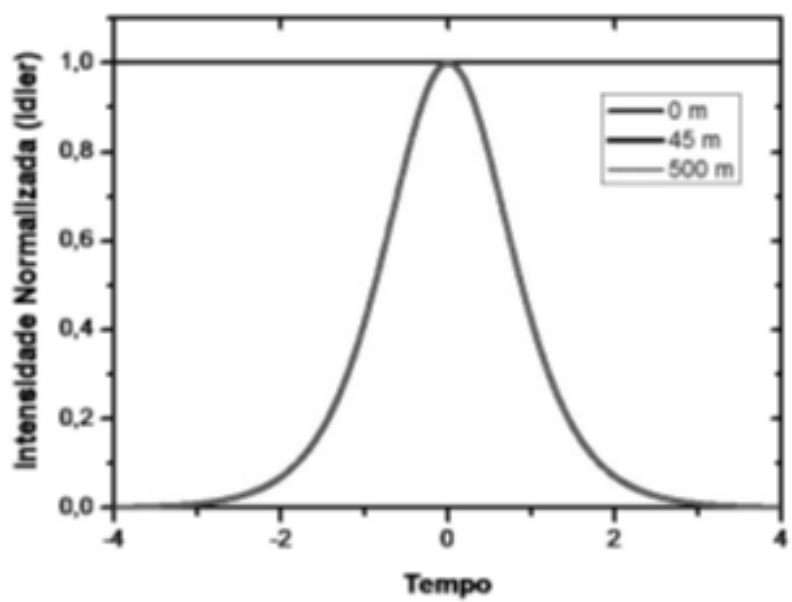

Fig. 3.4: Amplificação do Idler (5ps)-Potência Normalizada. 


\section{Conclusões}

O desenvolvimento desse trabalho proporcionou o conhecimento do comportamento de ENL em sinais ópticos em diversos tipos de guias de onda, mais especificamente, a propagação de pulsos curtos em sistemas WDM [5]. Além disso, observamos que com a utilização e o desenvolvimento de pulsos curtos ampliam-se as possibilidades de pesquisa não somente nessa direção, como também para outros dispositivos e efeitos não lineares. Mediante o exposto, percebemos que investimentos nesse ramo das telecomunicações trarão uma melhoria significativa nos sistemas de comunicações.

\section{Agradecimentos}

Agradecemos ao programa PIBIC-POLI pelo apoio financeiro essencial para realização desse trabalho.

\section{Referências}

[1] Govind P. Agawal. Nonlinear Fiber Optics. Academic Press, $3^{\text {a }}$ ed., San Diego, 2001

[2] Robert W. Boyd. Nonlinear Optics. Academic Press, San Diego, 1992.

[3] João F. L. Freitas, Amplificadores Ópticos, Estudos de Polarização e Geração de Pulsos Usando Processos Paramétricos em Fibras Ópticas. Tese de Doutorado, Departamento de Física - Universidade Federal de Pernambuco, Recife/Pe, 2007.

[4] Govind P. Agawal, Applications of Nonlinear Fiber Optics, Academic Press, San Diego, 2001.

[5] Govind P. Agawal, Lightwave Technology, Telecommunication Systems. Jonh Wiley \& Sons, Inc.,2005.

[6] I. Tomkos, I. Zacharopoulos, E. Roditi, D. Syvridis, and A. Uskov. Mechanisms of wave mixing and polarization sensitivity of the wavelength conversion in semiconductor optical amplifiers using two parallel polarized pumps. Opt. Commun., 163(1):49-54, 1999.

[7] Qiang Lin and Govind P. Agrawal. Vector theory of four wave mixing: polarization effects in fiberoptic parametric amplifiers. J. Opt. Soc. Am. B, 21(6):1216-1224, 2004.

[8] R. H. Stolen, E. P. Ippen, and A. R. Tynes. Vector theory offour wave mixing: polarization effects in fiber-optic parametric amplifiers. Appl. Phys. Lett.,20:62, 1972.

[9] Thomas Torounids. Fiber Optic Parametric Amplifiers in Single and Multi Wavelength Applications. PhD thesis, Department of Micro technology and Nanoscience, Chalmers University of Technology, Göteborg, Sweden, 2006.
[10] Jonas Hansryd, Peter A. Andrekson, Mathias Westlund, Jie Li, and Per-Olof Hedekvist. Fiberbased optical parametric amplifiers and their applications, IEEE J. Selet. Top. Quantum electron., 8(3):506-520, 2002.

[11] João F. L. Freitas, M. B. Costa e Silva, S. R. Lüthi, and A. S. L. Gomes. Raman enhanced parametric amplifier based $S-C$ band wavelength converter: Experiment and simulations. Opt. Commun., 255:314-318, 2005.

[12] S. R. Lüthi, G. F. Guimarães, B.B. C. Kyotoku, J. F. L. de Freitas, M. B. Costa e Silva, A. S. L. Gomes, B. H. Thomas, and J-P von der Weid. Optical Amplifier Hybrid Devises for S-band Telecommunications - A Comparison. Optical Amplifiers and Their Applications Conference, page JWB33, 2006.

[13] G. F. Guimarães, S. R. Lüthi, J. F. L. Freitas, and A. S. L. Gomes. TDFA/FOPA hybrid of broadband amplification and frequency conversion in optical communications. Elet. Lett., 42(17):997998, 2006. 